Case Report

\title{
Entamoeba dispar: A Rare Case of Enteritis in a Patient Living in a Nonendemic Area
}

\author{
Rosalia Graffeo, Carola Maria Archibusacci, Silvia Soldini, \\ Lucio Romano, and Luca Masucci
}

Institute of Microbiology, Catholic University of the Sacred Heart, Policlinico "Agostino Gemelli",

Largo Agostino Gemelli, 8-00168 Rome, Italy

Correspondence should be addressed to Lucio Romano; lucio.romano@rm.unicatt.it

Received 19 November 2013; Accepted 8 January 2014; Published 13 February 2014

Academic Editors: E. Altintas, N. Koide, and C. Vogt

Copyright (C) 2014 Rosalia Graffeo et al. This is an open access article distributed under the Creative Commons Attribution License, which permits unrestricted use, distribution, and reproduction in any medium, provided the original work is properly cited.

Entamoeba dispar, a common noninvasive parasite, is indistinguishable in its cysts and trophozoite forms from Entamoeba histolytica, the cause of invasive amebiasis, by microscopy. To differentiate the two species seems to be a problem for laboratory diagnosis. Recent experimental studies showed that E. dispar can be considered pathogenic too. We present a rare case of enteritis due to E. dispar.

\section{Introduction}

Entamoeba histolytica and Entamoeba dispar are two distinct but morphologically identical species living in the human colon $[1,2]$.

E. histolytica causes amebiasis. Amebiasis is one of the most common causes of death from protozoan parasitic disease, second only to malaria, with approximately 50 million cases and 100000 deaths annually, as reported by the WHO [3] and in areas where invasive amebiasis is common; E. dispar is by far the more prevalent species [4].

Until a few years ago, several studies distinguished between infections caused by E. histolytica, with invasive intestinal and extraintestinal disease, and those by E. dispar and E. moshkovskii, which were not considered pathogenic [5].

Recent studies showed E. dispar trophozoites to produce focal lesions in experimental animal models and to have lytic activity in cultured monolayer epithelial cells [6].

In 2012 Dolabella et al. described E. dispar trophozoites from the ICB-ADO strain (zymodeme I-nonpathogenic), inoculated intrahepatically in hamsters, which produced amoebic liver abscess [7].

Antigen detection, culture, and polymerase chain reaction are employed to distinguish E. histolytica from E. dispar. We report a case of enteritis due to E. dispar.

\section{Case Report}

An Italian 81-year-old woman suffered of abdominal pain and chronic diarrhea. She evacuated unformed stools three times a day for 10 days. She had not been hospitalized in the last year nor had she traveled to tropical countries.

Blood biochemistry and liver function tests were normal, and she was serologically negative for human immunodeficiency virus (HIV). Multiple stool cultures for bacterial pathogens, including Salmonella, Shigella, and Campylobacter, enterotoxigenic and other pathogenic E. coli and $C$. difficile toxin A/B were negative.

Stools collected for parasites were negative for ova and larvae by microscopy and for Giardia intestinalis and Cryptosporidium parvum by immunochromatographic test (CerTest Biotec S.L. Zaragoza-Spain).

Entamoeba histolytica/dispar/moshkovskii cysts were detected by microscopy at wet smear preparation with a $400 \mathrm{x}$ phase-contrast objective (Figure 1).

Culture was performed in medium based on Boeck and Drbohlav formulation (DiaSys Entamoeba kit, DiaSys Europe Ltd; Wokingham, UK).

We also used DNA-based methods to speciate E. histolytica, E. dispar, and E. moshkovskii as previously described [8-10]. 


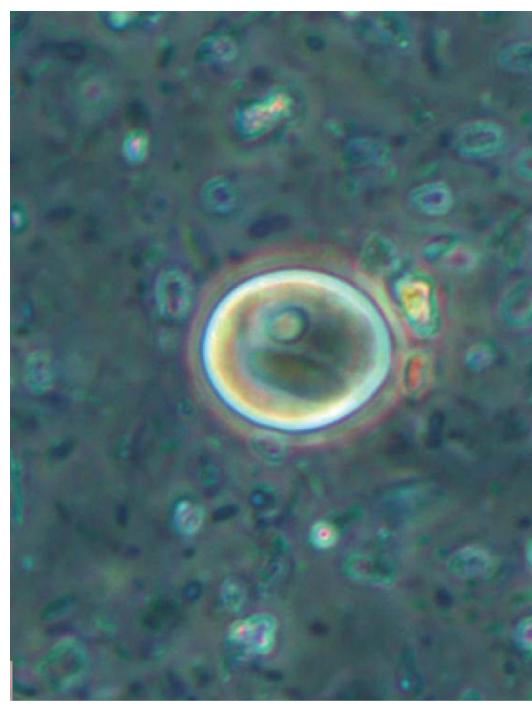

Figure 1: Entamoeba histolytica/dispar/moshkovskii cyst (original magnification 400x).

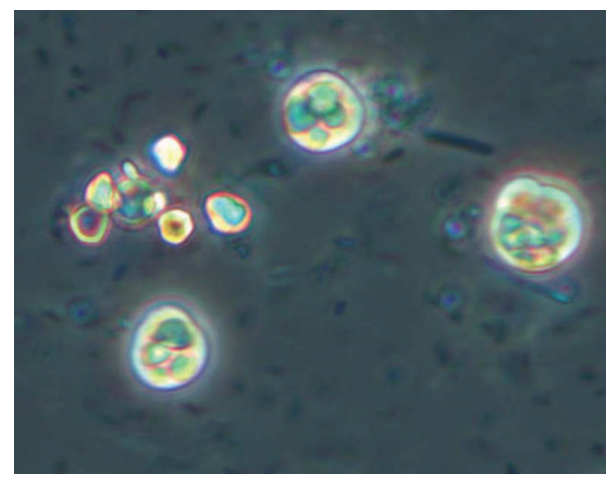

Figure 2: Entamoeba dispar trophozoites (molecular typing) in Boeck and Drbohlav formulation medium (original magnification 400x).

DNA was extracted from the clinical sample using the QIAamp DNA Mini Kit (Qiagen) according to manufacturer's instructions.

The nested multiplex polymerase chain reaction (PCR) [10], performed on stool, showed the species-specific product size for E. dispar (174 bp).

After 2 days, typical E. histolytica trophozoites were visualized in culture by microscopy (Figure 2). $[8,9]$.

PCR was performed on culture using two more protocols

By these methods, PCR products were specific for $E$. dispar too. The DNA amplicons were analyzed using the sequencing kit ABI PRISM dye terminator cycle and the automatic sequencing system ABI 3100 (Applied Biosystems), confirming E. dispar (GenBank accession number AB282661.1, genes for 18S rRNA, ITS1, 5.8S rRNA, ITS2).

We established a treatment protocol the same as luminal amebiasis (Paromomycin $30 \mathrm{mg} / \mathrm{kg} /$ day for 10 days in 3 divided doses). Patient responded promptly with resolution of diarrhea in 2 days.
Thirty days later, we used microscopy and PCR to test six samples of stool collected over a fourteen-day period. All samples were negative for E. histolytica/dispar.

\section{Conclusion}

Microscopy is still the gold standard to detect Entamoeba spp. in stool samples, but the use of routine diagnostic methods such as concentration and trichrome staining may be insufficient to demonstrate the presence of E. histolytica/dispar [11]. Studies about fecal antigen detection for pathogenic E. histolytica provide sensitivities and specificities that range from 87 to $97.6 \%$ and 92.6 to $98 \%$, respectively [11]. However PCRbased methods revealed greater sensitivity and specificity than ELISA (enzyme-linked immunosorbent assay) stool antigen detection kits, which employ monoclonal antibodies for the detection of E. histolytica and E. histolytica/dispar and that cannot discriminate between these genetically distinct organisms [12].

To distinguish E. histolytica and E. dispar, various molecular methods can be used [8-10].

Cultivation of E. histolytica plays a minor role, because this test is difficult, expensive, and labor-intensive to be routinely used in the diagnostic laboratory [13].

Culture techniques for Entamoeba spp. include xenic (diphasic and monophasic) and axenic systems. Culture of $E$. dispar is reported, but only in axenic or monoxenic medium $[14,15]$; protozoa isolated from the patient in this study has grown in a xenic culture.

Xenic cultivation is defined as the growth of the parasite in the presence of an undefined bacteria flora, while monoxenic culture occurs in the presence of a specific bacterium. The xenic culture of E. histolytica was first introduced by Boeck and Drbohlav in 1925 in a diphasic egg slant medium, and a modification of this medium (Locke-egg) is still used today. Purified rice starch is important for growth of $E$. histolytica as a carbohydrate source in xenic cultivation. In fact soluble sugars cannot be used because they would be metabolized rapidly by the bacteria [13]. Within 1-4 days of incubation at $37^{\circ} \mathrm{C}$ in this medium, E. histolytica trophozoites appear with characteristic hyaline ectoplasm with engulfed starch crystal, according to manufacturer's instructions.

E. dispar in humans was always considered a commensal organism. Using an animal model, it has also been demonstrated that this species causes tissue lesions in the intestine and serious damage to epithelial cells $[16,17]$.

The spectrum of clinical manifestations observed widely varies among individuals infected with $E$. dispar, ranging from amoebic liver abscess production [18] to symptomatic nondysenteric colitis [6]. Some authors even recommend not to treat the latter infection [19].

The patient observed in this study was investigated for bacterial pathogens and parasites. Stool samples analyzed were positive only for E. dispar. In this case, therapy was decisive, so the patient could completely recover.

Other intestinal parasites such as Cyclospora cayetanensis were always considered pathogens for humans in endemic areas and linked to travellers and immunocompromised 
patients, but cases of immunocompetents and no-travel relations are reported [20]. Infections due to protozoa are acquired by the fecal-oral route through ingestion of infective oocysts or cysts in contaminated water or on contaminated fruits and vegetables, which today, in the "globalization era," is easy to observe everywhere [21].

Like in this report, diseases and pathogens are constantly evolving and for this reason to the question of Ximénez et al. "Human amebiasis: breaking the paradigm?" we answer "YES."

\section{Conflict of Interests}

The authors declare that there is no conflict of interests regarding the publication of this paper.

\section{References}

[1] L. S. Diamond and C. G. Clark, "A redescription of Entamoeba histolytica Schaudinn, 1903 (Emended Walker, 1911) separating it from Entamoeba dispar Brumpt, 1925," The Journal of Eukaryotic Microbiology, vol. 40, no. 3, pp. 340-344, 1993.

[2] R. Haque, I. K. M. Ali, S. Akther, and W. A. Petri Jr., "Comparison of PCR, isoenzyme analysis, and antigen detection for diagnosis of Entamoeba histolytica infection," Journal of Clinical Microbiology, vol. 36, no. 2, pp. 449-452, 1998.

[3] WHO, "Amoebiasis," The Weekly Epidemiological Record, vol. 72, pp. 97-100, 1997.

[4] V. Gathiram and T. F. H. G. Jackson, "Frequency distribution of Entamoeba histolytica zymodemes in a rural South African population," The Lancet, vol. 1, no. 8431, pp. 719-721, 1985.

[5] S. Katzwinkel-Wladarsch, T. Loscher, and H. Rinder, "Direct amplification and differentiation of pathogenic and nonpathogenic Entamoeba histolytica DNA from stool specimens," American Journal of Tropical Medicine and Hygiene, vol. 51, no. 1, pp. 115-118, 1994.

[6] A. O. Costa, M. A. Gomes, O. A. Rocha, and E. F. Silva, "Pathogenicity of Entamoeba dispar under xenic and monoxenic cultivation compared to a virulent E. histolytica," Revista do Instituto de Medicina Tropical de Sao Paulo, vol. 48, no. 5, pp. 245-250, 2006.

[7] S. S. Dolabella, J. Serrano-Luna, F. Navarro-García et al., "Amoebic liver abscess production by entamoeba dispar," Annals of Hepatology, vol. 11, no. 1, pp. 107-117, 2012.

[8] P. Gonin and L. Trudel, "Detection and differentiation of Entamoeba histolytica and Entamoeba dispar isolates in clinical samples by PCR and enzyme-linked immunosorbent assay," Journal of Clinical Microbiology, vol. 41, no. 1, pp. 237-241, 2003.

[9] C. G. Clark and L. S. Diamond, "Ribosomal RNA genes of "pathogenic" and "nonpathogenic" Entamoeba histolytica are distinct," Molecular and Biochemical Parasitology, vol. 49, no. 2, pp. 297-302, 1991.

[10] K. Khairnar and S. C. Parija, "A novel nested multiplex polymerase chain reaction (PCR) assay for differential detection of Entamoeba histolytica, E. moshkovskii and E. dispar DNA in stool samples," BMC Microbiology, vol. 7, article 47, 2007.

[11] L. S. Garcia, R. Y. Shimizu, and C. N. Bernard, "Detection of Giardia lamblia, Entamoeba histolytica/Entamoeba dispar, and Cryptosporidium parvum antigens in human fecal specimens using the triage parasite panel enzyme immunoassay," Journal of Clinical Microbiology, vol. 38, no. 9, pp. 3337-3340, 2000.
[12] D. Mirelman, Y. Nuchamowitz, and T. Stolarsky, "Comparison of use of enzyme-linked immunosorbent assay-based kits and PCR amplification of rRNA genes for simultaneous detection of Entamoeba histolytica and E. dispar," Journal of Clinical Microbiology, vol. 35, no. 9, pp. 2405-2407, 1997.

[13] C. G. Clark and L. S. Diamond, "Methods for cultivation of luminal parasitic protists of clinical importance," Clinical Microbiology Reviews, vol. 15, no. 3, pp. 329-341, 2002.

[14] C. G. Clark, "Axenic cultivation of Entamoeba dispar Brumpt 1925, Entamoeba insolita Geiman and Wichterman 1937 and Entamoeba ranarum Grassi 1879," The Journal of Eukaryotic Microbiology, vol. 42, no. 5, pp. 590-593, 1995.

[15] S. Kobayashi, E. Imai, H. Tachibana, T. Fujiwara, and T. Takeuchi, "Entamoeba dispar: cultivation with sterilized Crithidia fasciculata," The Journal of Eukaryotic Microbiology, vol. 45, no. 2, pp. 3S-8S, 1998.

[16] M. Espinosa-Cantellano, A. González-Robles, B. Chávez et al., "Entamoeba dispar: ultrastructure, surface properties and cytopathic effect," The Journal of Eukaryotic Microbiology, vol. 45, pp. 265-272, 1998.

[17] M. Espinosa-Cantellano, G. Castañón Gutiérrez, and A. Martínez-Palomo, "In vivo pathogenesis of Entamoeba dispar," Archives of Medical Research, vol. 28, supplement, pp. S204S206, 1997.

[18] C. Ximénez, R. Cerritos, L. Rojas et al., "Human amebiasis: breaking the paradigm?" International Journal of Environmental Research and Public Health, vol. 7, no. 3, pp. 1105-1120, 2010.

[19] N. Pestehchian, M. Nazary, A. Haghighi, M. Salehi, and H. Yosefi, "Frequency of Entamoeba histolytica and Entamoeba dispar prevalence among patients with gastrointestinal complaints in Chelgerd city, southwest of Iran," Journal of Research in Medical Sciences, vol. 16, no. 11, pp. 1436-1440, 2011.

[20] L. Masucci, R. Graffeo, M. Siciliano, A. Franceschelli, F. Bugli, and G. Fadda, "First Italian case of Cyclosporiasis in an immunocompetent woman: local acquired infection," New Microbiologica, vol. 31, no. 2, pp. 281-284, 2008.

[21] L. Masucci, R. Graffeo, S. Bani et al., "Intestinal parasites isolated in a large teaching hospital, Italy," Eurosurveillance, vol. 16, no. 24, 2011. 


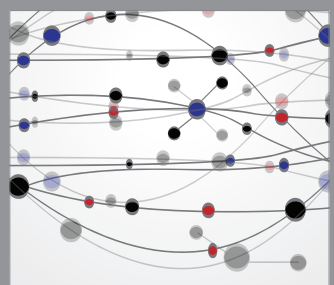

The Scientific World Journal
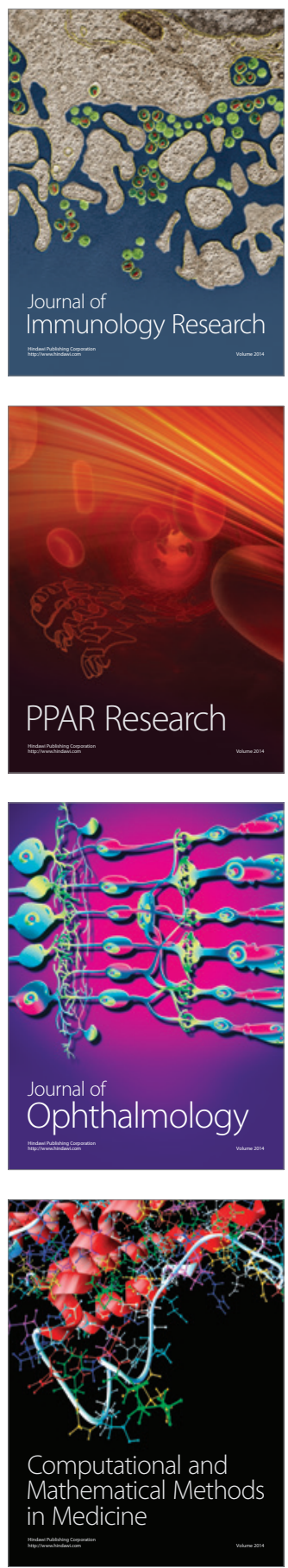

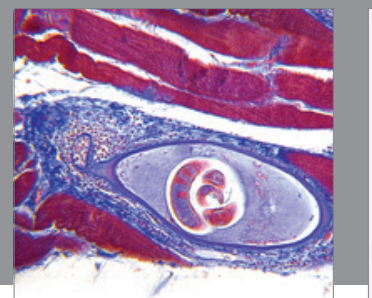

Gastroenterology

Research and Practice
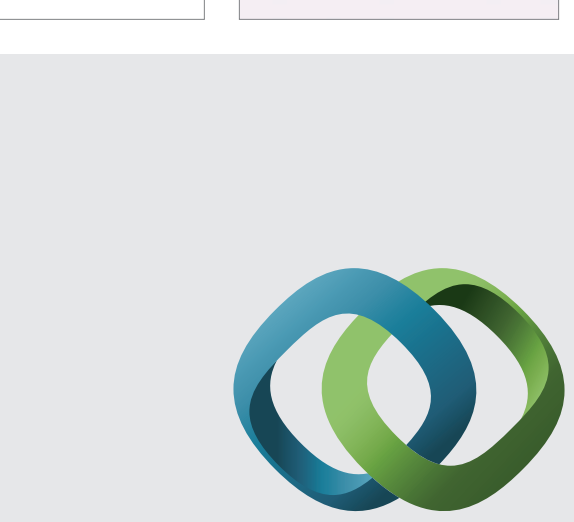

\section{Hindawi}

Submit your manuscripts at

http://www.hindawi.com
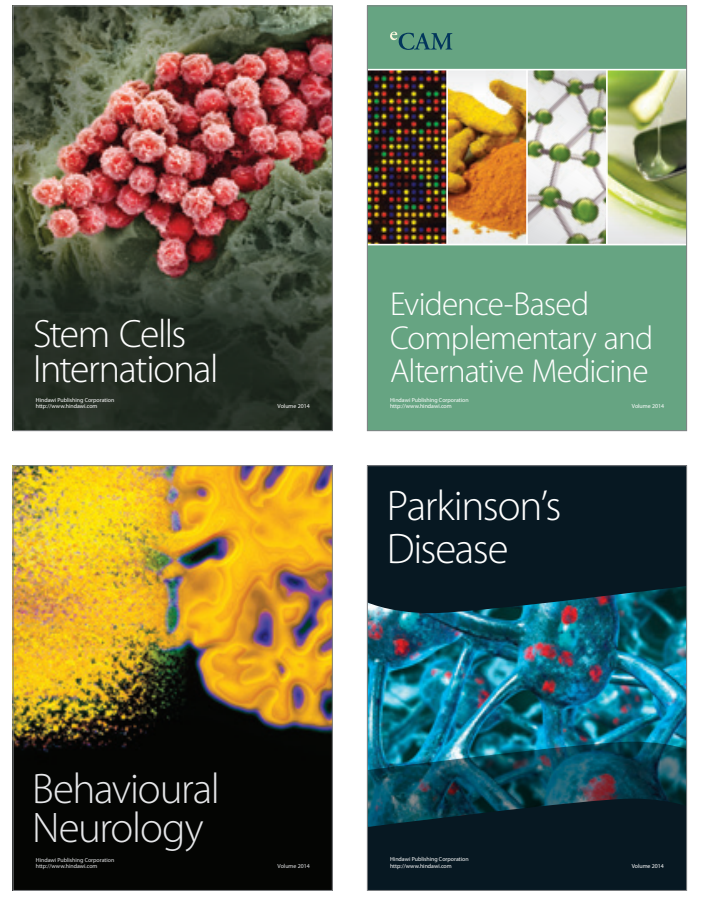
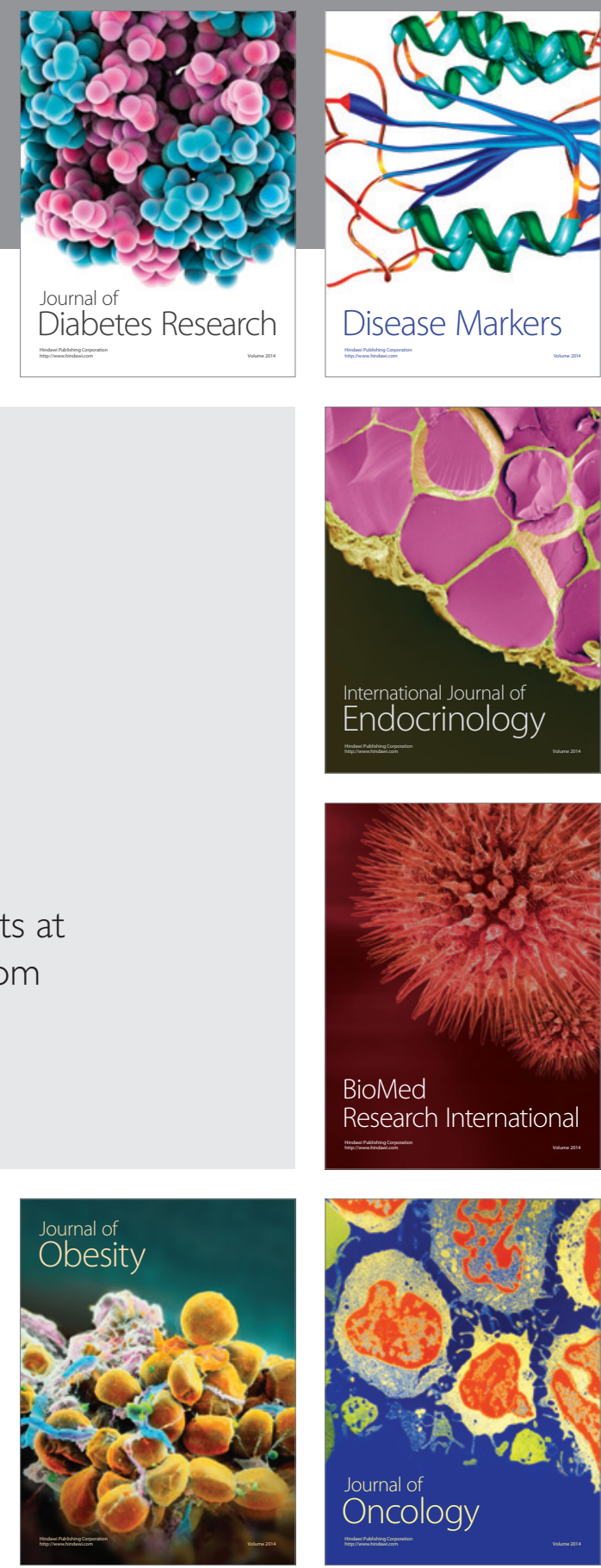

Disease Markers
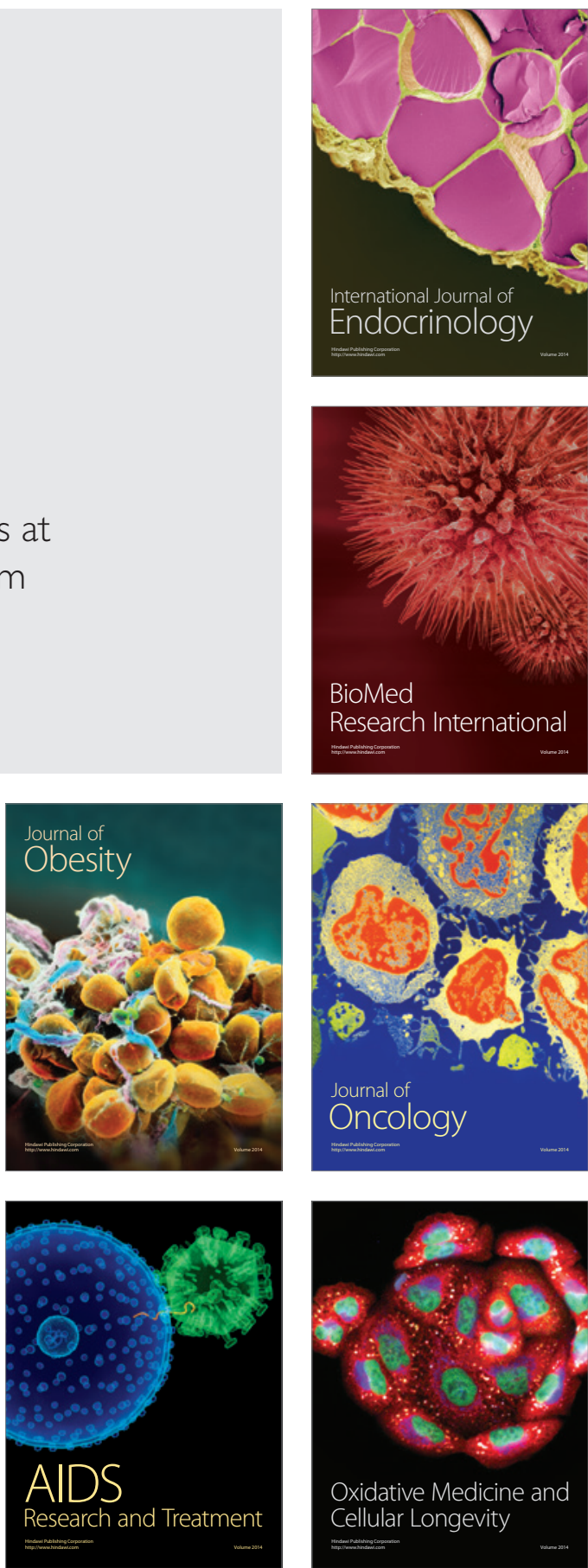